\title{
Spectroscopic analysis and docking simulation on the recognition and binding of TEM-1 $\beta$-lactamase with $\beta$-lactam antibiotics
}

\author{
JIANTING YANG ${ }^{1,2}$, QIAN LI ${ }^{1}$ and LIUJIAO BIAN ${ }^{1}$ \\ ${ }^{1}$ Department of Traditional Chinese Medicine, College of Life Science, Northwest University, Xi'an, \\ Shaanxi 710069; ${ }^{2}$ Drug and Equipment Department, Weapon Industry 521 Hospital, Xi'an, Shaanxi 710065, P.R. China
}

Received April 23, 2016; Accepted March 17, 2017

DOI: $10.3892 /$ etm.2017.4853

\begin{abstract}
The interaction between TEM-1 $\beta$-lactamase and antibiotics is very important in the hydrolysis of antibiotics. In the present study, the recognition and binding of TEM-1 $\beta$-lactamase with three $\beta$-lactam antibiotics, including penicillin $\mathrm{G}$, cefalexin and cefoxitin, was investigated by fluorescence and ultraviolet-visible absorption spectra in combination with molecular docking in the temperature range of 278-288 K and under simulated physiological conditions. The results demonstrated that the fluorescence emissions of TEM-1 $\beta$-lactamase were extinguished by static quenching and the energy of TEM-1 $\beta$-lactamase was transferred in a non-radioactive manner. The binding of TEM-1 $\beta$-lactamase with the three antibiotics was a spontaneously exothermic process, with binding constants of $1.41 \times 10^{7}, 7.81 \times 10^{6}$ and $5.43 \times 10^{4}$ at $278 \mathrm{~K}$. Furthermore, binding was driven by enthalpy change and the binding forces between them were mainly hydrogen bonding and Van der Waals forces. A TEM-1 $\beta$-lactamase only bound with one antibiotic at a time and the binding capacity between them was closely associated with the functional groups and flexibility in the antibiotics. In addition, a conformational change occurred in the TEM-1 $\beta$-lactamases when they bound with the three antibiotics and TEM-1 $\beta$-lactamase-antibiotic complexes were formed. The present study provided an insight into the recognition and binding of TEM-1 $\beta$-lactamase with $\beta$-lactam antibiotics, which may be helpful for designing a novel substrate for TEM-1 $\beta$-lactamase and developing novel antibiotics that are resistant to the enzyme.
\end{abstract}

\section{Introduction}

Some superbugs accurately catalyze the hydrolysis of the $\beta$-lactam ring in $\beta$-lactam antibiotics through their target enzyme, TEM-1 $\beta$-lactamases, and thereby lead to the antibiotics

Correspondence to: Professor Liujiao Bian, Department of Traditional Chinese Medicine, College of Life Science, Northwest University, 229 Taibai Road, Xi'an, Shaanxi 710069, P.R. China E-mail: bianliujiao@sohu.com

Key words: TEM-1 $\beta$-lactamase, $\beta$-lactam antibiotics, binding, fluorescence spectroscopy, molecular docking losing efficacy against them (1). The recognition and binding of TEM-1 $\beta$-lactamase with the antibiotics is a precondition for the hydrolysis of the antibiotics (2). Furthermore, $\beta$-lactam antibiotics have been widely used as therapeutic agents in the treatment of bacterial infections. These antibiotics share a structural characteristic, a four-membered $\beta$-lactam ring, and are able to irreversibly bind to the active sites of penicillin-binding proteins, thus inhibiting these bacterial proteins from synthesizing cell walls and leading to cell death $(3,4)$. However, the widespread overuse of $\beta$-lactam antibiotics, such as in clinical treatment and animal farming, has resulted in the emergence of antibiotic-resistant bacteria, which has become a serious worldwide clinical issue (5). Various bacteria have developed effective defense mechanisms against $\beta$-lactam antibiotics by producing $\beta$-lactamases, which are able to effectively catalyze the hydrolysis of the $\beta$-lactam ring in $\beta$-lactam antibiotics (6-8).

Based on sequence similarity, $\beta$-lactamase has been subdivided into four classes: Classes A-D (9). $\beta$-lactamases belonging to classes A, C and D are active-site serine enzymes, and those belonging to class $\mathrm{B}$ require a metal (typically $\mathrm{Zn}^{2+}$ ) for their catalytic activity. The class A TEM family is one of the most commonly identified plasmid-mediated $\beta$-lactamase families within Gram-negative bacteria (10). Through the deprotonation of the side-chain $\mathrm{OH}$ group, the Ser70 residue of the TEM $\beta$-lactamase in the active site is activated by Glu166 to form Ser70- $\Omega$-loop, which subsequently opens the $\beta$-lactam ring through acylation with the $\beta$-lactam carbonyl group $(11,12)$. TEM-1 $\beta$-lactamase is predominantly produced by Escherichia coli that are resistant to ampicillin, and is regarded as the ancestor of the TEM family (13). Furthermore, TEM-1 $\beta$-lactamase has strong hydrolysis ability against penicillin and first generation cephalosporin (14). When the TEM-1 $\beta$-lactamase catalyzes the hydrolysis of the $\beta$-lactam ring in $\beta$-lactam antibiotics, the first step is the recognition and binding of the enzyme with antibiotics, accompanied by structural change (14). Subsequently, hydrolysis of the $\beta$-lactam antibiotics and expulsion of the products occurs (14). The former is a physical process and is necessary preparation for the latter chemical process.

However, there is little research on the recognition and binding between TEM-1 $\beta$-lactamase and antibiotics. A study by Wang et al (15) demonstrated structural insight into the kinetics and DeltaCp of interactions between TEM-1 $\beta$-lactamase and $\beta$-lactamase inhibitory protein 
(BLIP). Furthermore, a study by Legendre et al (16) used TEM-1 $\beta$-lactamase as a scaffold for protein recognition and assays. They demonstrated that TEM-1 $\beta$-lactamase was useful in replacement of enzyme-conjugated antibodies in enzyme-linked immunosorbent assays or in other applications that utilize enzyme-conjugated antibodies. A study by Hanes et al (17) explored the specificity and cooperativity of $\beta$-lactamase at position 104 in TEM-1/BLIP and SHV-1/BLIP interactions and established a quantitative understanding of the determinants of affinity in protein-protein interactions. Additionally, a study by Cheong et al (18) utilized TEM-1 $\beta$-lactamase with wild-type activity as a rapid drug sensor for in vitro drug screening, and developed a novel fluorescent drug sensor through the combined strategy of Val216 $\rightarrow$ Cys216 mutation and fluorophore labeling. Furthermore, a study by Phichith et al (19) demonstrated that the tryptophan residue of Pep90 was of crucial importance for its inhibitory activity to both TEM-1 $\beta$-lactamase and penicillin-binding proteins. Through engineering allosteric regulation, a study by Mathieu et al (20) demonstrated that the binding of a ligand to the hinge region of TEM-1 $\beta$-lactamase was able to modify the positioning of hinge regions and modulate their activities.

Developing novel and potent $\beta$-lactam antibiotics insensitive to TEM-1 $\beta$-lactamases is extremely important in view of the clinical threat that we currently face (21). Fluorescence spectra are selective, highly sensitive and easy to operate when analyzing conformational changes of enzyme molecules, and microenvironment changes of amino acid residues (22). Ultraviolet-visible (UV-Vis) absorption spectra have been demonstrated to have advantages when investigating the quenching mechanism of the enzyme-ligand complex, allowing for the binding constant and the number of binding sites to be obtained (23). Therefore, the aim of the present study was to explore the recognition and binding of $\beta$-lactamase with $\beta$-lactam antibiotics. In order to do this, TEM-1 $\beta$-lactamase was selected as a model enzyme and three typical $\beta$-lactam antibiotics, including penicillin $\mathrm{G}$, cefalexin and cefoxitin, were selected as model antibiotics. Recognition and binding investigations were performed using fluorescence and UV-Vis absorption spectra in combination with molecular docking under simulated physiological conditions. The present study may provide a guideline for the research and development of novel antibiotics that are resistant to TEM-1 $\beta$-lactamase enzymes.

\section{Materials and methods}

Reagents. TEM-1 $\beta$-lactamase was purchased from Alpha Diagnostic International, Inc., (San Antonio, TX, USA). Penicillin $G$, cefalexin and cefoxitin were purchased from Sigma-Aldrich (Merck KGaA, Darmstadt, Germany) and used without further purification. $\mathrm{Na}_{2} \mathrm{HPO}_{4}$ and $\mathrm{NaH}_{2} \mathrm{PO}_{4}$ were purchased from Tianjin Kermel Chemical Reagent Co., Ltd., (Tianjin, China). Solutions of antibiotics and TEM-1 $\beta$-lactamase were prepared in phosphate buffer solution $(0.020 \mathrm{~mol} / \mathrm{l} ; \mathrm{pH} 7.0)$ and were kept in the dark in a refrigerator. Other chemicals were analytical grade reagents and double-distilled water was used throughout the study.

Instruments. Fluorescence measurements were performed with a Hitachi Model F-4500 spectrofluorometer (Hitachi,
Ltd., Tokyo, Japan) equipped with a $150 \mathrm{~W}$ xenon lamp (Hitachi, Ltd., Tokyo, Japan). All absorption spectra were obtained on a UV-2550 dual-beam UV-Vis spectrophotometer (Hitachi, Ltd.). The $\mathrm{pH}$ values of each solution were measured using a pHS-3C pH meter (Shanghai Rex Instrument Factory, Shanghai, China) equipped with a combined glass electrode. The FA-2004 precision electronic balance was from Mettler Toledo (Zurich, Switzerland).

$U V$-Vis absorption measurements. At $278 \mathrm{~K}$, various concentrations of penicillin $\mathrm{G}$, cefalexin and cefoxitin solutions were added to TEM-1 $\beta$-lactamase solution $\left(5 \times 10^{-6} \mathrm{~mol} / \mathrm{l}\right)$. The concentrations of the three antibiotics were gradually increased from 0 to $25 \times 10^{-6} \mathrm{~mol} / 1$. Following mixing and interaction for $2 \mathrm{~min}$, the UV-Vis absorption spectra were recorded on a spectrophotometer with a slit of $2 \mathrm{~nm}$ and scanning speed of $400 \mathrm{~nm} / \mathrm{min}$ using $0.02 \mathrm{~mol} / \mathrm{l}$ phosphate buffer ( $\mathrm{pH} 7.0$ ) as a reference. The scanning range was set to $200-400 \mathrm{~nm}$.

Fluorescence emission measurements. The fluorescence emission measurements were performed at temperatures of 278 , 283 and $288 \mathrm{~K}$. The excitation wavelength was separately set at 278 and $295 \mathrm{~nm}$. Furthermore, the concentration of TEM-1 $\beta$-lactamase solution was stabilized at $2.5 \times 10^{-7} \mathrm{~mol} / 1$, and the content of penicillin $\mathrm{G}$ varied between 0 and $11.25 \times 10^{-8} \mathrm{~mol} / \mathrm{l}$ at intervals of $1.25 \times 10^{-8} \mathrm{~mol} / \mathrm{l}$. Additionally, the excitation and emission slit widths were all set at $10 \mathrm{~nm}$. In total, $0.02 \mathrm{~mol} / \mathrm{l}$ phosphate buffer ( $\mathrm{pH}$ 7.0) was subtracted to correct background fluorescence. Synchronous fluorescence spectra were also recorded with increasing concentrations of antibiotics by setting wavelength difference $(\Delta \lambda)=60 \mathrm{~nm}$ for tryptophan residues and $\Delta \lambda=15 \mathrm{~nm}$ for tyrosine residues. Furthermore, the excitation slit width was $5 \mathrm{~nm}$ and the emission slit width was $10 \mathrm{~nm}$.

The inner filter effect was estimated according to the following equation:

$$
\mathrm{F}_{\text {corr }}=\mathrm{F}_{\mathrm{obs}} \times 10^{\frac{\mathrm{A}_{\mathrm{cx}} \cdot \mathrm{d}_{\mathrm{cx}}}{2}+\frac{\mathrm{A}_{\mathrm{cm}} \cdot \mathrm{d}_{\mathrm{cm}}}{2}}
$$

In this equation, $\mathrm{F}_{\text {corr }}$ was the correct fluorescence intensity that would be measured in the absence of inner-filter effects, $F_{\text {obs }}$ was the measured fluorescence, and $d_{e x}$ and $d_{e m}$ were the cuvette path length in the excitation and emission direction (in $\mathrm{cm})$, respectively. $A_{e x}$ and $A_{e m}$ were the measured change in absorbance value at the excitation and emission wavelengths, respectively, caused by ligand addition (in a $1-\mathrm{cm}$ path length cuvette) (24).

Determination of potassium iodide (KI) quenching of the fluorescence spectrum. At $278 \mathrm{~K}$, ten TEM-1 $\beta$-lactamase solutions $\left(2.5 \times 10^{-7} \mathrm{~mol} / \mathrm{l}\right)$ were prepared. Following this, a certain volume of KI solution, which contained $2.0 \mathrm{mmol} / 1 \mathrm{Na}_{2} \mathrm{~S}_{2} \mathrm{O}_{3}$ as the reductant, was separately added to the above solutions and their fluorescence emission intensities were measured. The concentration of KI added in solution was increased gradually from $0.01-0.09 \mathrm{~mol} / 1$, and the TEM-1 $\beta$-lactamase solution in the absence of KI was used as a blank control.

Molecular docking. The known crystal structure of TEM-1 $\beta$-lactamase [Protein Data Bank (PDB) code, 1ZG4] was downloaded from the RCSB PDB (www.rcsb.org/pdb). 

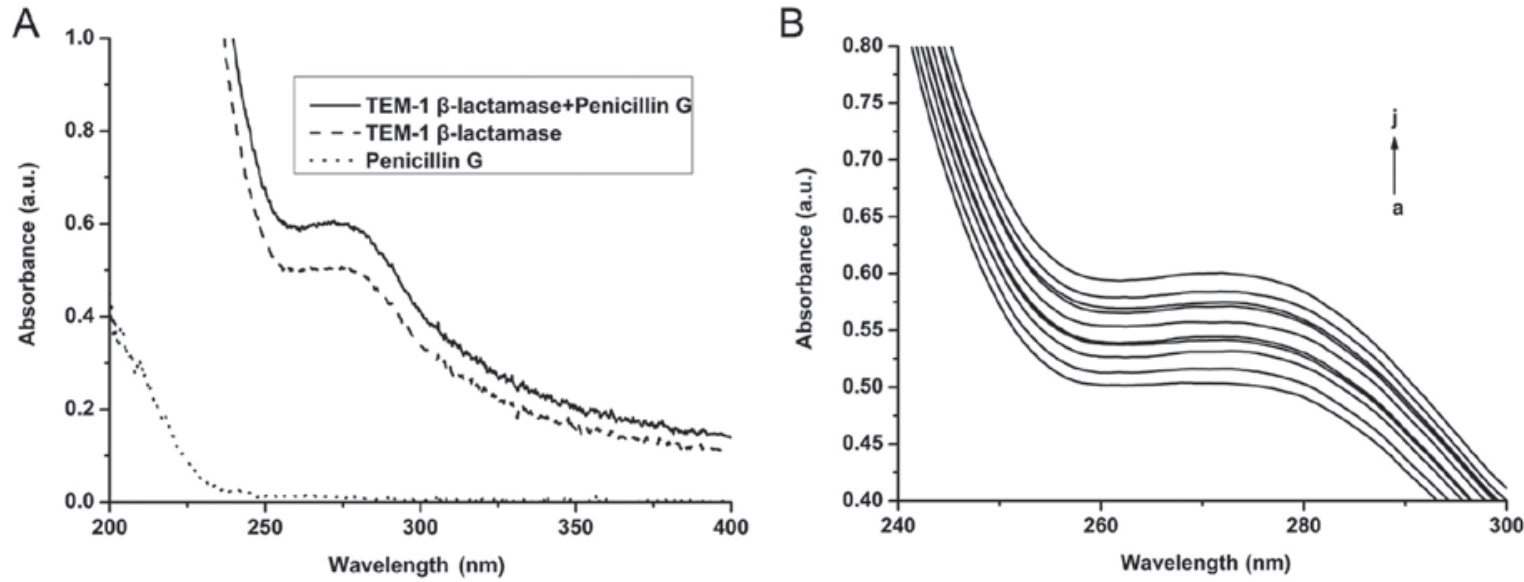

Figure 1. (A) UV-Vis absorption spectra of penicillin G, TEM-1 $\beta$-lactamase and the penicillin G-TEM-1 complexes. (B) UV-Vis absorption spectra of TEM-1 $\beta$-lactamase in the presence of various concentrations of penicillin $\mathrm{G} . \mathrm{C}_{\mathrm{TEM}-\mathrm{l}}=5.0 \mathrm{x} 10^{-6} \mathrm{~mol} / \mathrm{l} ; \mathrm{C}_{\mathrm{penicillin}} \mathrm{G}(\mathrm{a}-\mathrm{j}): 0,1.0,2.0,3.0,4.0,5.0,10.0,15.0,20.0$ and $25.0 \times 10^{-6} \mathrm{~mol} / 1$. UV-Vis, ultraviolet-visible.

Pretreatments, including the deletion of water molecules and heteroatoms, and the addition of hydrogen atoms, were performed using the Molecular Operating Environment v. 2008.10 (MOE; Chemical Computing Group, Inc., Montreal, QC, Canada). Three-dimensional structures of the antibiotics were built using ChemDraw Ultra v. 8.0 (CambridgeSoft; PerkinElmer, Inc., Waltham, MA, USA). The minimized energy structures of antibiotics were obtained by MOE and the resultant geometries were read in AutoDock v. 4.2 software (Scripps Research Institute, La Jolla, CA, USA) in a compatible file format, from which the required files were generated in AutoDock 4.2. The semi-flexible docking process was adopted in the initial rigid treatment of TEM-1 $\beta$-lactamase and freely rotating antibiotic molecules, as described in a previously published study (25). Furthermore, molecular docking was conducted using the AutoDock v. 4.2 suite of programs, which utilizes the Lamarckian Genetic Algorithm, a hybrid of a genetic algorithm with an adaptive local search (26). The lowest binding energy conformation was determined out of 50 different conformations for each docking simulation, and the resultant minimum energy conformation was applied for further analysis. PyMOL 1.5.0.3 (Schrödinger, LLC, New York, NY, USA) and MOE software packages were then used for the visualization of the docked conformations $(27,28)$.

\section{Results and Discussion}

Binding of TEM-1 $\beta$-lactamase with the three antibiotics $U V$-Vis absorption. In the presence of various concentrations of penicillin G, the UV-Vis absorption spectra of TEM-1 $\beta$-lactamase were recorded at $278 \mathrm{~K}$ (Fig. $1 \mathrm{~A}$ and $\mathrm{B}$ ). As demonstrated in Fig. 1A, the UV-Vis absorption spectrum of the penicillin G-TEM-1 system was markedly different to that of penicillin $\mathrm{G}$ and TEM-1 $\beta$-lactamase, indicating the formation of new complexes between penicillin $\mathrm{G}$ and TEM-1 $\beta$-lactamase (29). As demonstrated in Fig. 1B, the UV-Vis absorption of TEM-1 $\beta$-lactamase increased and a slight red-shift occurred as the concentration of penicillin $G$ increased, indicating that the interaction between penicillin $G$ and TEM-1 $\beta$-lactamase resulted in subtle conformational changes of TEM-1 $\beta$-lactamase. Similar results were also observed in the UV-Vis absorption spectra of the cefalexin- and cefoxitin-TEM-1 systems (data not shown). A hyperchromic effect and slight red-shift of the maximum absorption peak were also separately observed with increasing cefalexin or cefoxitin concentrations in solution (data not shown). These results indicated that the three $\beta$-lactam antibiotics were all able to bind with TEM-1 $\beta$-lactamase and form stable complexes.

Fluorescence quenching. The fluorescence quenching by three antibiotics contributed to the study of the mechanism of the interaction between enzymes and antibiotics and allowed for the calculation of binding parameters. As demonstrated in Fig. 2A and B, the fluorescence intensity of TEM-1 $\beta$-lactamase decreased with the increase in penicillin $G$ concentration, although no marked changes occurred in the peak shape and position. The emission of TEM-1 $\beta$-lactamase peaked at $342 \mathrm{~nm}$, whereas the emission of penicillin $\mathrm{G}$ peaked at $270 \mathrm{~nm}$ (data not shown). Furthermore, the fluorescence emission of penicillin $\mathrm{G}$ was very weak, meaning that there was no interference in the fluorescence spectroscopies of penicillin G and TEM-1 $\beta$-lactamase. Additionally, the fluorescence emission of TEM-1 $\beta$-lactamase was also demonstrated to be excited at a wavelength of $278 \mathrm{~nm}$, and it was constantly higher than that at a wavelength of $295 \mathrm{~nm}$ (Fig. 2). This result is consistent with the fact that tryptophan, tyrosine and phenylalanine residues are all fluorogenic at $278 \mathrm{~nm}$, whereas only the tryptophan residues are fluorogenic at $295 \mathrm{~nm}(30)$.

The intensity of protein fluorescence may be decreased by a wide variety of processes (31). Such decreases in intensity are called quenching. It may be inferred that penicillin $\mathrm{G}$ may bind to and interact with TEM-1 $\beta$-lactamase and form a new complex, eventually leading to the fluorescence quenching of TEM-1 $\beta$-lactamase (32). Similar fluorescence quenching was also observed in the presence of cefalexin and cefoxitin (data not shown).

Fluorescence quenching data are usually analyzed by the Stern-Volmer equation (33) shown below:

$$
\text { (i) } \frac{\mathrm{F}_{0}}{\mathrm{~F}}=1+K_{\mathrm{SV}} \cdot[\mathrm{Q}]=1+k_{\mathrm{q}} \cdot \tau_{0} \cdot[\mathrm{Q}] \text {, }
$$



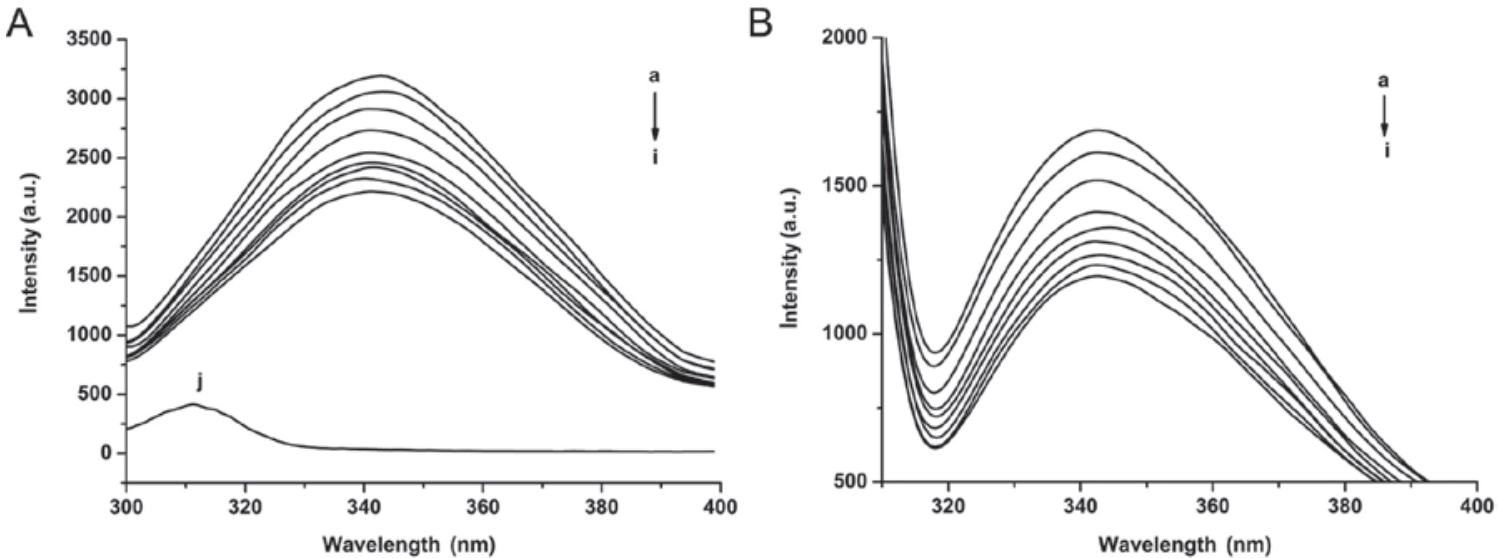

Figure 2. Fluorescence spectra of TEM-1 $\beta$-lactamase with the addition of various concentrations of penicillin $\mathrm{G}$ at (A) $\lambda_{\mathrm{ex}}=278 \mathrm{~nm}$ and (B) $\lambda_{\mathrm{ex}}=295 \mathrm{~nm}$. $\mathrm{C}_{\mathrm{TEM}-\mathrm{l}}=5.0 \times 10^{-6} \mathrm{~mol} / 1 ; \mathrm{C}_{\text {penicillin G }}(\mathrm{a}-\mathrm{i}): 0,1.25,2.50,3.75,5.00,6.25,8.75,10.00$ and $11.25 \times 10^{-8} \mathrm{~mol} / 1 . \mathrm{j}$, penicillin G: $11.25 \times 10^{-8}$ mol/1. $\lambda_{\text {ex }}$, excitation wavelength

where $\mathrm{F}_{0}$ and $\mathrm{F}$ are the steady-state fluorescence intensities in the absence and presence of a quencher, respectively, $K_{\mathrm{Sv}}$ is the Stern-Volmer quenching constant, [Q] is the concentration of the quencher, $k_{\mathrm{q}}$ is the bimolecular quenching rate constant and $\tau_{0}$ is the lifetime of the fluorophores in the enzyme molecules in the absence of quenchers, which is $10^{-8} \mathrm{sec}$ for large biomolecules, such as proteins and enzymes (33).

After drawing the Stern-Volmer fitted curve of TEM-1 $\beta$-lactamase with penicillin $\mathrm{G}$ at 278 (Fig. 3), and the other two similar fitted curves at 283 and $288 \mathrm{~K}$, the $K_{\mathrm{SV}}$ and $k_{\mathrm{q}}$ parameters were derived from the slope of equation (i), as demonstrated in Table I. The $K_{\mathrm{Sv}}$ values gradually decreased with increasing temperature for all three antibiotics and the rate constants $k_{\mathrm{q}}$ were all greater than the collisional quenching rate constant of biological macromolecules $\left(2.0 \times 10^{10} \mathrm{l} / \mathrm{mol} / \mathrm{s}\right)(34)$. Therefore, TEM-1 $\beta$-lactamases experienced a static quenching by the three antibiotics.

Binding constant and the number of binding sites. Fluorescence quenching of TEM-1 $\beta$-lactamases by the three antibiotics demonstrated static quenching, and the following equation may be used to further derive two characteristic parameters, the binding constant $\left(K_{\mathrm{a}}\right)$ and the number of binding sites per TEM-1 (n) (35):

$$
\text { (ii) } \lg \frac{\mathrm{F}_{0}-\mathrm{F}}{\mathrm{F}}=\lg K_{\mathrm{a}}+n \cdot \lg [\mathrm{Q}] \text {. }
$$

According to equation (ii), the linear fittings of $\log \left[\left(\mathrm{F}_{0}-\mathrm{F}\right) / \mathrm{F}\right]$ vs. $\log [\mathrm{Q}]$ were able to be performed at 278,283 and $288 \mathrm{~K}$. The linear fitting at $278 \mathrm{~K}$ is demonstrated in Fig. 4. Following this, the two characteristic parameters, $K_{\mathrm{a}}$ and $n$, were separately derived from the intercepts and slopes of these linear plots, and are demonstrated in Table II. The results indicated that as the temperature increased from 278 to $288 \mathrm{~K}$, the $K_{a}$ of penicillin $\mathrm{G}$ decreased from $1.41 \times 10^{7}$ to $7.62 \times 10^{6}$, and the number of binding sites was $\sim 1$ at all temperatures. These observations indicated that penicillin $\mathrm{G}$, cephalexin and cefoxitin were able to bind to TEM- $1 \beta$-lactamase at only one binding site, and that penicillin $\mathrm{G}$ had the highest binding affinity to TEM-1 $\beta$-lactamase, followed by cefalexin and cefoxitin, respectively. In addition, the decrease in $K_{a}$ with increasing temperature suggested that the temperature

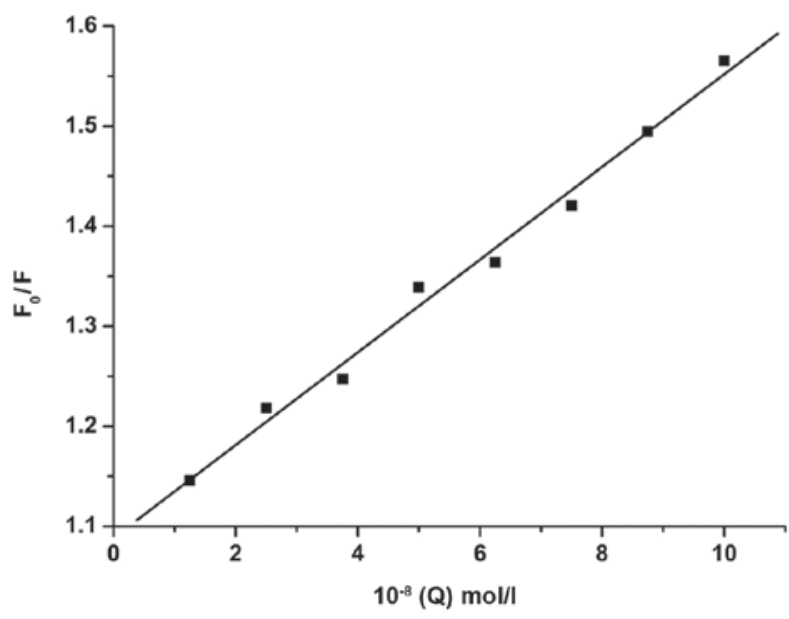

Figure 3. Stern-Volmer curve of TEM-1 $\beta$-lactamase with penicillin $G$ at $278 \mathrm{~K} . \mathrm{F}_{0}$, steady-state fluorescence intensity in the absence of a quencher; $\mathrm{F}$, steady-state fluorescence intensity in the presence of a quencher; Q, concentration of the quencher

change affected the binding of these antibiotics to TEM-1 $\beta$-lactamase, with weaker binding affinities associated with higher temperatures.

Thermodynamic data and binding force. Whether the binding of enzymes with their ligands is able to occur spontaneously, in theory, depends on the free energy change $(\Delta G)$ of the system (36). It is an advantage to spontaneous binding when $\Delta G$ is negative. During the binding of enzymes with their ligands, there are four types of non-covalent interactions: Hydrogen bonds, hydrophobic interactions, electrostatic interactions and Van der Waals forces (36). Furthermore, the binding form may be identified based on the values of enthalpy changes $(\Delta H)$ and entropy changes $(\Delta S)$. According to the Ross law, $\Delta H>0$ and $\Delta S>0$ indicates that the main interaction force is a hydrophobic interaction, $\Delta H<0$ and $\Delta S>0$ indicates that the main interaction force is electrostatic interaction, and $\Delta H<0$ and $\Delta \mathrm{S}<0$ indicates that the main interaction forces are hydrogen bond interaction and Van der Waals forces (36). Therefore, it is possible to determine the binding force types between TEM- $1 \beta$-lactamase and 
Table I. Stern-Volmer quenching parameters of TEM-1 $\beta$-lactamase with penicillin G, cefalexin and cefoxitin at three different temperatures.

Antibiotic

\begin{tabular}{llccr} 
& \multicolumn{1}{c}{ Pemperature, $\mathrm{K}$} & Penicillin $\mathrm{C}$ & Cefalexin & Cefoxitin \\
\hline 278 & $K_{\mathrm{SV}} \times 10^{6}, 1 / \mathrm{mol}$ & 4.63 & 5.62 & 5.41 \\
& $k_{\mathrm{q}} \times 10^{14}, 1 / \mathrm{mol} / \mathrm{sec}$ & 4.63 & 5.62 & 5.41 \\
283 & $K_{\mathrm{Sv}} \times 10^{6}, 1 / \mathrm{mol}$ & 3.94 & 5.08 & 4.51 \\
& $k_{\mathrm{qx}} 10^{14}, 1 / \mathrm{mol} / \mathrm{sec}$ & 3.94 & 5.08 & 4.51 \\
288 & $K_{\mathrm{SV}} \times 10^{6}, 1 / \mathrm{mol}$ & 2.47 & 4.59 & 3.15 \\
& $k_{\mathrm{qx}} 10^{14}, 1 / \mathrm{mol} / \mathrm{sec}$ & 2.47 & 4.59 & 3.15 \\
\hline
\end{tabular}

$K_{\mathrm{SV}}$, Stern-Volmer quenching constant; $k_{\mathrm{q}}$, bimolecular quenching rate constant.

Table II. $K_{\mathrm{a}}$ values and $n$ of TEM- $1 \beta$-lactamase with penicillin G, cefalexin and cefoxitin at three different temperatures.

\begin{tabular}{lcccc}
\hline & & \multicolumn{3}{c}{ Antibiotic } \\
\cline { 3 - 5 } Temperature, $\mathrm{K}$ & Parameters & Penicillin $\mathrm{C}$ & Cefalexin & Cefoxitin \\
\hline 278 & $K_{\mathrm{a}}, 1 / \mathrm{mol}$ & $1.41 \times 10^{7}$ & $7.81 \times 10^{6}$ & $5.43 \times 10^{4}$ \\
& $n$ & 1.06 & 1.03 & 0.69 \\
283 & $K_{\mathrm{a}}, 1 / \mathrm{mol}$ & $1.09 \times 10^{7}$ & $6.46 \times 10^{6}$ & $1.07 \times 10^{4}$ \\
& $n$ & 1.06 & 1.02 & 0.60 \\
288 & $K_{\mathrm{a}}, 1 / \mathrm{mol}$ & $7.62 \times 10^{6}$ & $1.97 \times 10^{6}$ & $8.96 \times 10^{3}$ \\
& $n$ & 1.05 & 0.94 & 0.60 \\
\hline
\end{tabular}

$K_{\mathrm{a}}$, binding constant; $n$, number of binding sites.

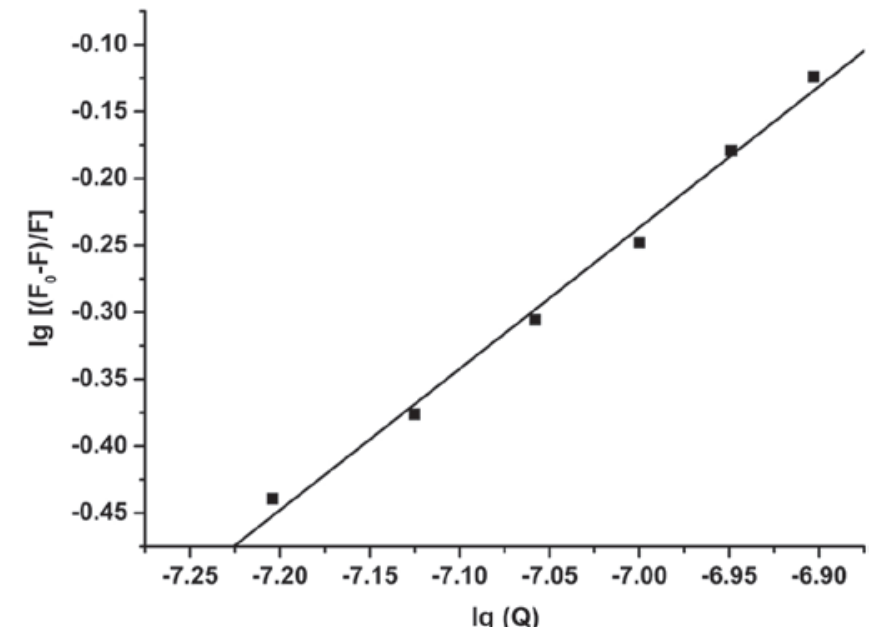

Figure 4. Plot of $\log \left[\left(\mathrm{F}_{0}-\mathrm{F}\right) / \mathrm{F}\right]$ vs. $\log [\mathrm{Q}]$ of TEM-1 $\beta$-lactamase with penicillin $\mathrm{G}$ at $278 \mathrm{~K}$. $\mathrm{F}_{0}$, steady-state fluorescence intensity in the absence of a quencher; F, steady-state fluorescence intensity in the presence of a quencher; $\mathrm{Q}$, concentration of the quencher.

antibiotics. The $\Delta H$ and $\Delta S$ may be derived by the following Van't Hoff equation:

$$
\ln K_{\mathrm{a}}=-\frac{\Delta H}{\mathrm{R} \cdot \mathrm{T}}+\frac{\Delta S}{\mathrm{R}}
$$

where $\mathrm{R}(8.314 \mathrm{~J} / \mathrm{K} / \mathrm{mol})$ is the gas constant, $\mathrm{T}$ is the experimental temperature and $K_{a}$ is the binding constant at the corresponding temperature (T). Therefore, $\Delta H$ and $\Delta S$ may be obtained from the intercept and slope of the linear plot of $\ln K_{a}$ vs. $1 / \mathrm{T}$, respectively. Additionally, $\Delta G$ may be further obtained using the following equation: $\Delta G=\Delta H-T \cdot \Delta S$.

Using the $K_{a}$ in Table II, the $\Delta H, \Delta S$ and $\Delta G$ for the binding of TEM-1 $\beta$-lactamases with the three antibiotics at three experimental temperatures were obtained (Table III). The results demonstrated that the $\Delta \mathrm{G}$ were all $<0$, suggesting that all the binding of the TEM-1 enzyme with the three antibiotics was spontaneous. Furthermore, all $\Delta H$ and $\Delta S$ values were $<0$, indicating that all binding between TEM-1 $\beta$-lactamase and the three antibiotics was driven by enthalpy change. Additionally, all binding forces between TEM- $1 \beta$-lactamase and the three antibiotics were predominantly hydrogen bonds and Van der Waals forces.

Apparent activation energy. A reactant molecule may reach its activated state only through direct effective collision (37). The lowest amount of energy that is required for an ordinary molecule to become an activated molecule with sufficient energy is called apparent activation energy (37). This apparent activation energy in the reaction process may be obtained through the Arrhenius equation, as follows: 
Table III. Thermodynamic data for the binding processes of TEM-1 $\beta$-lactamase with penicillin G, cefalexin and cefoxitin .

\begin{tabular}{lccrr}
\hline & & \multicolumn{3}{c}{ Antibiotic } \\
\cline { 3 - 5 } Thermodynamic parameter & Temperature & Penicillin $\mathrm{G}$ & Cefalexin & Cefoxitin \\
\hline$\Delta H, \mathrm{~kJ} / \mathrm{mol}$ & - & -42.26 & -95.63 & -124.71 \\
$\Delta S, \mathrm{~J} / \mathrm{mol} / \mathrm{K}$ & - & -14.75 & -209.91 & -359.08 \\
$\Delta G, \mathrm{~kJ} / \mathrm{mol}$ & 278 & -38.16 & -37.25 & -24.89 \\
& 283 & -38.09 & -36.12 & -23.09 \\
& 288 & -38.01 & -35.15 & -21.29 \\
\hline
\end{tabular}

$\Delta H$, enthalpy changes; $\Delta S$, entropy changes; $\Delta G$, free energy change.

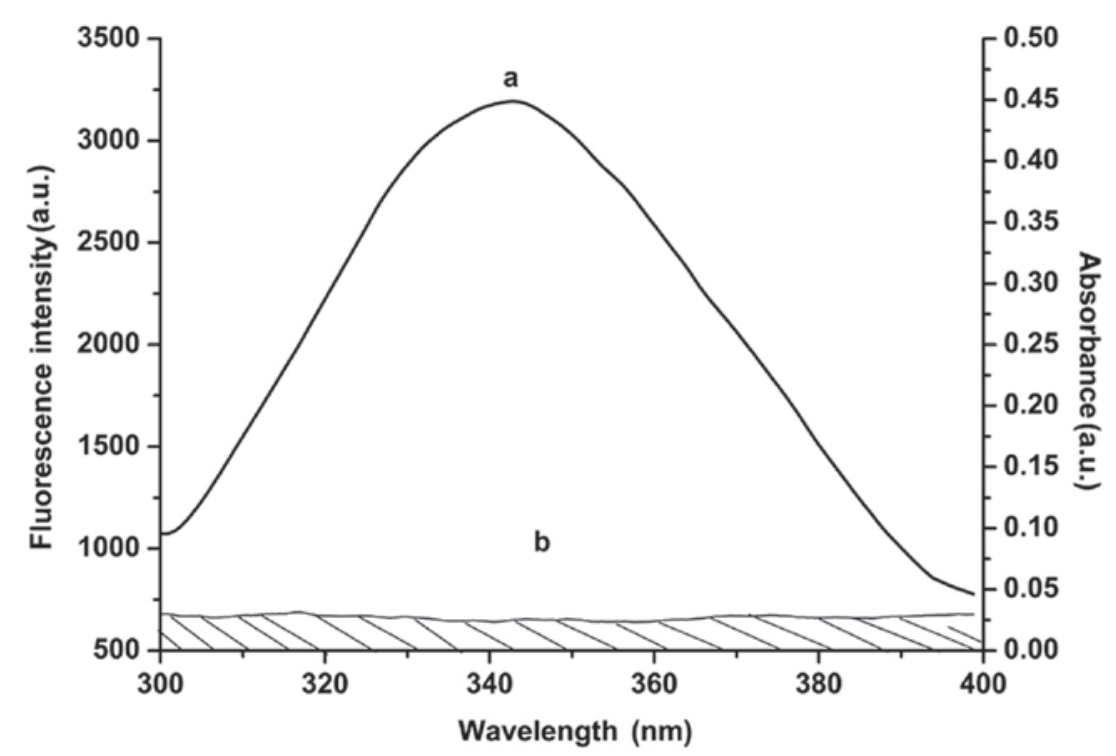

Figure 5. Overlapped area between the (a) fluorescence emission spectrum of TEM-1 $\beta$-lactamase and (b) the UV-Vis absorption spectrum of penicillin G. UV-Vis, ultraviolet-visible.

$$
\text { (iii) } k_{\mathrm{q}}=\mathrm{A} \cdot \mathrm{e}^{\frac{-\Delta E_{\mathrm{a}}}{\mathrm{RT}}}
$$

In this equation, $\mathrm{A}$ is the pre-exponential factor, $\Delta E_{\mathrm{a}}$ is the apparent activation energy and $k_{q}$ is the reaction rate constant at reaction temperature (RT). The following equation may be obtained by taking the logarithm on both sides of equation (iii):

$$
\ln k_{\mathrm{q}}=\ln \mathrm{A}-\frac{\Delta E_{\mathrm{a}}}{\mathrm{RT}}
$$

Therefore, through a linear plot of $\ln k_{q}$ vs. 1/T, it is possible to derive $\Delta E_{\mathrm{a}}$ from the slope of the line.

The fluorescence quenching rate constants, $k_{q}$, of TEM-1 $\beta$-lactamase by three antibiotics at 278,283 and $288 \mathrm{~K}$ were introduced into the above equation, and $\Delta E_{\mathrm{a}}$ values of penicillin $\mathrm{G}$, cefalexin and cefoxitin were estimated as $-43.65,-33.96$ and $-37.41 \mathrm{~kJ} / \mathrm{mol}$, respectively. The $\Delta E_{\mathrm{a}}$ values of the three binding systems were all negative, suggesting that there was no energy barrier for the binding processes of TEM- $1 \beta$-lactamase with the three antibiotics, and that the binding molecules did not require energy to transform from the non-activated to the activated state.
Reaction kinetics may be used to identify reaction mechanisms, predominantly by investigating the details of a reaction process, such as the reaction rate and activation energy. The most fundamental issue with regard to reaction kinetics is involved with the activation energy, which is the difference between the energy of an activated molecule and that of an ordinary (non-activated) molecule in the reaction process (37).

Energy transfer and binding distance. According to the Förster energy transfer theory, dipole-dipole energy transfer occurs in a non-radioactive form when two types of biomolecules interact with each other, and the excitation energy is transferred from a fluorescent donor to a fluorescent acceptor through electrostatic interactions (38). This process is determined by three main factors. Firstly, the fluorescent donor (TEM-1 $\beta$-lactamase) must fluoresce. Secondly, the fluorescence spectrum of the fluorescent donor must partially overlap with the UV absorption spectrum of the fluorescent acceptor (antibiotics). Lastly, the donor and acceptor must interact with each other in a binding distance $<7 \mathrm{~nm}$ (38). The energy transfer efficiency (E) may be calculated as follows: 
Table IV. Binding distance for the binding of TEM-1 $\beta$-lactamase with penicillin $\mathrm{G}$, cefalexin and cefoxitin.

Parameter

\begin{tabular}{lccc}
\cline { 2 - 4 } Antibiotic & $\mathrm{E}$ & $\mathrm{R}_{0}, \mathrm{~nm}$ & $r, \mathrm{~nm}$ \\
\hline Penicillin $\mathrm{G}$ & 0.104 & 1.45 & 2.08 \\
Cefalexin & 0.092 & 1.43 & 2.09 \\
Cefoxitin & 0.085 & 1.41 & 2.10
\end{tabular}

E, energy transfer efficiency; $\mathrm{R}_{0}$, critical distance for $50 \%$ energy transfer efficiency; $r$, binding distance between the donor and the acceptor.

$$
\text { (iv) } \mathrm{E}=\frac{\mathrm{R}_{0}{ }^{6}}{\mathrm{R}_{0}{ }^{6}+r^{6}}=1-\frac{\mathrm{F}}{\mathrm{F}_{0}}
$$

where $r$ is the binding distance between the donor and the acceptor and $\mathrm{R}_{0}$ is the critical distance for $50 \%$ energy transfer efficiency.

In order to determine $\mathrm{R}_{0}{ }^{6}$, the following equation may be used (v) $\mathrm{R}_{0}^{6}=8.8 \times 10^{-25} \mathrm{x}\left(K^{2} \cdot N^{4} \cdot \phi \cdot \mathrm{J}\right)$. In this equation, $K^{2}$ is the space factor of orientation, $N$ is the refracted constant of medium, $\phi$ is the fluorescence quantum yield of the donor, and $J$ is the effect of the spectral overlap between the emission spectrum of the donor and the UV absorption spectrum of the acceptor (Fig. 5). These parameters may be determined using the following equation:

$$
\text { (vi) } J=\frac{\Sigma \mathrm{F}(\lambda) \cdot \varepsilon(\lambda) \cdot \lambda^{4} \cdot \Delta \lambda}{\Sigma \mathrm{F}(\lambda) \cdot \Delta \lambda}
$$

where $F(\lambda)$ is the fluorescence intensity of the donor in the wavelength range $\lambda-(\lambda+\Delta \lambda), \varepsilon(\lambda)$ is the extinction coefficient of the acceptor at $\lambda$. E may be obtained by using equation (iv).

The following equation is also required:

$$
\text { (vii) } \mathrm{A}=\lg \left(\frac{1}{\mathrm{D}}\right)=\varepsilon(\lambda) \cdot \mathrm{b} \cdot \mathrm{c}
$$

where $\mathrm{A}$ is the absorbance at wavelength $\lambda, \mathrm{D}$ is the transmittance, namely the ratio of the intensity of the transmitted light to that of the incident light, $b$ is the thickness of the light-absorbing medium, and $\mathrm{c}$ is the concentration of the light-absorbing material $(39,40)$.

In the present case, $K_{2}=2 / 3, \mathrm{~N}=1.336$ and $\phi=0.118$. According to equations (iv)-(vii), $\mathrm{J}, \mathrm{R}_{0}, \mathrm{E}$ and $r$ may be calculated, as demonstrated in Table IV. The average distance $r<8 \mathrm{~nm}$ and $0.5 \mathrm{R}_{0}<r<1.5 \mathrm{R}_{0}$, indicated that there was a high probability of energy transfer from TEM-1 $\beta$-lactamase to three antibiotics in a non-radioactive manner when the three antibiotics bound to TEM-1 $\beta$-lactamase.

\section{Conformational changes of TEM-1 $\beta$-lactamase}

Fluorescence quenching by KI. Fluorescence quenching by KI was implemented for the investigation of chromophoric residues on the surface of TEM-1-antibiotic complexes. KI possesses strong polarity, and it only acts on the chromophoric amino acids on the surface of an enzyme molecule, while exerting no effect on the hydrophobic region inside the enzyme molecule (41).
When a series of different concentrations of KI solution were added into the TEM- $1 \beta$-lactamase or TEM-1-antibiotic complex system, fluorescence quenching was analyzed by the modified Stern-Volmer equation, as follows:

$$
\text { (viii) } \frac{\mathrm{F}_{0}}{\mathrm{~F}_{\mathrm{o}}-\mathrm{F}}=\frac{1}{f_{\mathrm{m}}}+\frac{1}{f_{\mathrm{m}} \cdot K_{\mathrm{MSV}}} \cdot \frac{1}{[\mathrm{Q}]},
$$

where $\mathrm{F}_{0}, \mathrm{~F}$ and $[\mathrm{Q}]$ are the same as in equation (i). $K_{\mathrm{MSV}}$ is the modified Stern-Volmer quenching constant and $f_{\mathrm{m}}$ is the fraction of fluorophore accessible to the quencher. Therefore, by utilizing the plot of $\mathrm{F}_{0} /\left(\mathrm{F}_{0}-\mathrm{F}\right)$ vs. [Q], the parameters, $K_{\mathrm{MSv}}$ and $f_{\mathrm{m}}$, were able to be separately obtained by the slope and intercept of equation (viii). In the present study, the concentration of TEM-1 $\beta$-lactamase was much lower than the concentration of quencher KI in the binding system. Therefore, the fluorescence quenching of TEM-1 $\beta$-lactamase and TEM-1-antibiotic complexes by $\mathrm{KI}$ was examined at $278 \mathrm{~K}$ and the parameters, $K_{\mathrm{MSV}}$ and $f_{\mathrm{m}}$, were determined according to equation (viii) (Table V).

The modified Stern-Volmer quenching constant, $K_{\mathrm{MSV}}$, is the reciprocal of the concentration of quencher when the fluorescence intensity of fluorophores is quenched to half of $\mathrm{F}_{0}$. Furthermore, a higher $K_{\mathrm{MSV}}$ indicates that the chromophoric residues on the surface of the enzyme molecule are more readily accessible to KI, leading to greater quenching $(42,43)$. The results in Table $\mathrm{V}$ demonstrated that, for the three antibiotics, all the quenching constants $K_{\mathrm{MSV}}$ of TEM-1-antibiotic complexes are greater than those of TEM-1 $\beta$-lactamase, indicating that the chromophoric residues on the surface of TEM-1-antibiotic complexes are more accessible to KI than those on the surface of the enzyme molecule. This means that a change occurs in the conformation of TEM- $1 \beta$-lactamase when it binds with antibiotics to form a TEM-1-antibiotic complex. Furthermore, the results in Table $\mathrm{V}$ demonstrated that, for the three antibiotics, the fractions of the TEM-1 fluorophores accessible to the quencher were all greater than those of the TEM-1-antibiotic fluorophores accessible to the quencher. Additionally, upon the formation of the TEM-1-penicillin G complex, the fraction of the fluorophores accessible to the quencher decreases by $22.0 \%$, whereas when cefalexin and cefoxitin bind with this enzyme, the fractions of the fluorophores accessible to the quencher are reduced by 13.1 and $5.4 \%$, respectively. These results indicate that a decreased fraction of the fluorophore is accessible to the quencher upon the binding of TEM- $1 \beta$-lactamase with the three antibiotics, and a conformational change takes place in TEM-1 $\beta$-lactamase when it binds with the three antibiotics to form TEM-1-antibiotic complexes. Additionally, it was further indicated that, of the three antibiotics, penicillin $\mathrm{G}$ exhibited the greatest capacity to alter the conformation of TEM-1 $\beta$-lactamase upon binding to the enzyme, whereas cefoxitin demonstrated the least capacity to change the conformation of TEM-1 $\beta$-lactamase.

Synchronous fluorescence. Synchronous fluorescence provides information directly related to the molecular environment in the vicinity of the chromosphere molecules (44). In the present study, when the $\mathrm{D}$-value $(\Delta \lambda)$ between the excitation wavelength and emission wavelength were stabilized at 15 or $60 \mathrm{~nm}$, the synchronous fluorescence gave the characteristic information of tyrosine or tryptophan residues (45). The effect of penicillin 
Table V. Effects of fluorescence quenching of TEM-1 $\beta$-lactamase and TEM-1-antibiotic complexes by potassium iodide.

\begin{tabular}{llccc}
\hline \multirow{2}{*}{ Antibiotic } & \multicolumn{2}{c}{ Parameter } \\
\cline { 3 - 4 } Penicillin G & Substrate & $K_{\mathrm{MSV}}, 1 / \mathrm{mol}$ & $f_{\mathrm{m}}$ & Reduction extent, $\%$ \\
& TEM-1 $\beta$-lactamase & $4.39 \times 10^{2}$ & 0.6303 & 22.0 \\
Cefalexin & TEM-1- penicillin complex & $4.67 \times 10^{2}$ & 0.4916 & 13.1 \\
& TEM-1 $\beta$-lactamase & $3.23 \times 10^{2}$ & 0.5778 & \multirow{2}{*}{5.4} \\
Cefoxitin & TEM-1-cefalexin complex & $3.61 \times 10^{2}$ & 0.5019 & \\
& TEM-1 $\beta$-lactamase & $3.24 \times 10^{2}$ & 0.4414 & 0.4177 \\
\hline
\end{tabular}

$K_{\mathrm{MSv}}$, modified Stern-Volmer quenching constant; $f_{\mathrm{m}}$, fraction of fluorophore accessible to the quencher.

A

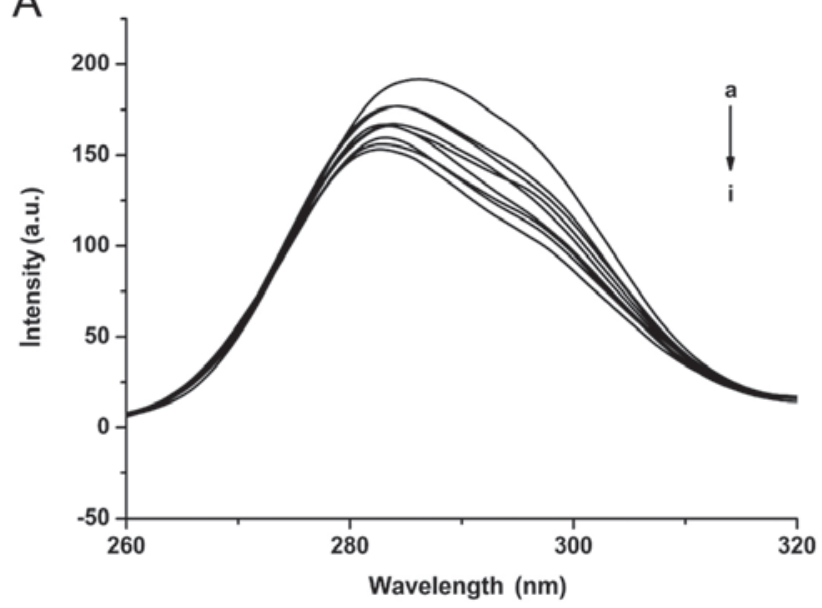

B

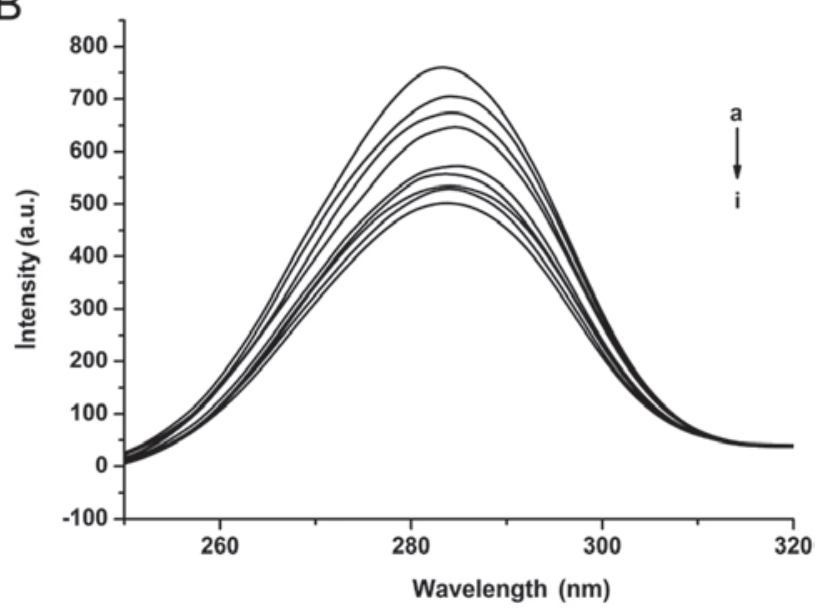

Figure 6. Synchronous fluorescence spectra of TEM-1 $\beta$-lactamase under various concentrations of penicillin G at (A) $\Delta \lambda=15 \mathrm{~nm}$ and (B) $\Delta \lambda=60 \mathrm{~nm}$. $\mathrm{C}_{\text {penicillin G }}(\mathrm{a}-\mathrm{i}): 0,1.25,2.50,3.75,5.00,6.25,8.75,10.00$ and $11.25 \times 10^{-8} \mathrm{~mol} / 1 . \Delta \lambda$, wavelength difference.
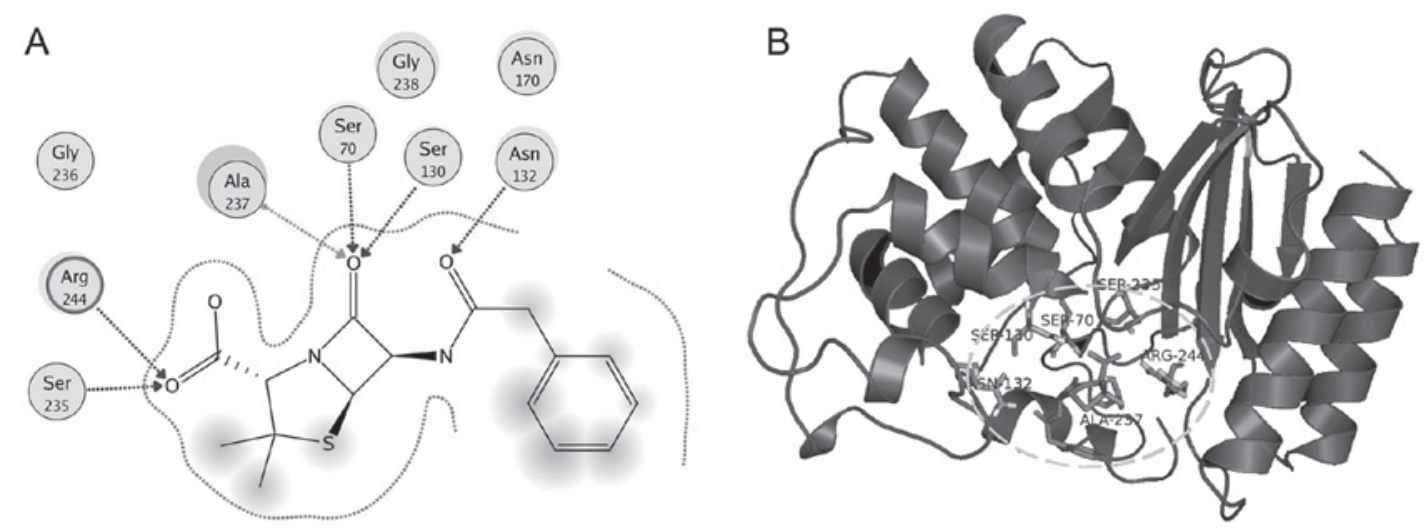

Figure 7. (A) Two-dimensional and (B) three-dimensional views of the molecular docking of TEM-1 $\beta$-lactamase with penicillin G.

G on TEM-1 $\beta$-lactamase synchronous fluorescence spectroscopy is demonstrated in Fig. 6. The fluorescence quenching degree when $\Delta \lambda=60 \mathrm{~nm}$ was markedly greater than that when $\Delta \lambda=15 \mathrm{~nm}$.

It is apparent from Fig. 6B that the maximum emission wavelength moderately shifts towards long wave when $\Delta \lambda=60 \mathrm{~nm}$. The shift effect indicated that the conformation of TEM-1 $\beta$-lactamase was altered when penicillin $\mathrm{G}$ bound to the enzyme. Furthermore, it also indicated that the polarity around the tryptophan residues was increased and the hydrophobicity was decreased. This result agrees with the conformational changes observed by UV-Vis spectra. Furthermore, similar results were observed in the binding of TEM-1 $\beta$-lactamases with the other two antibiotics, cefalexin and cefoxitin. Therefore, it may be concluded that a conformational change occurs in TEM-1 $\beta$-lactamases when they 
Table VI. Energies of the binding complexes of TEM-1 $\beta$-lactamase with penicillin $\mathrm{G}$, cefalexin and cefoxitin.

\begin{tabular}{lcccc}
\hline & \multicolumn{4}{c}{ Energy parameter, kJ/mol } \\
\cline { 2 - 5 } Antibiotic & $\Delta G$ & $\Delta E_{1}$ & $\Delta E_{2}$ & $\Delta E_{3}$ \\
\hline Penicillin G & -3.68 & -4.88 & -4.78 & -0.13 \\
Cefalexin & -2.62 & -5.30 & -4.63 & -0.67 \\
Cefoxitin & -2.30 & -3.49 & -3.12 & -0.39 \\
\hline
\end{tabular}

$\Delta G$, binding energy change in the binding process, which is calculated in water solvent using a scoring function; $\Delta E_{1}$, intermolecular interaction energy, which is a sum of Van der Waals, hydrogen bonding, desolvation free and electrostatic energies; $\Delta E_{2}$, sum of Van der Waals, hydrogen bonding and desolvation free energies; $\Delta E_{3}$, electrostatic energy.

bind with the three antibiotics to form TEM-1-antibiotic complexes.

Molecular docking. The binding of the three antibiotics to TEM-1 $\beta$-lactamases was simulated by molecular docking. Fig. 7A and $\mathrm{B}$ demonstrate the modeling diagram of the molecular docking for the binding of penicillin $\mathrm{G}$ to the TEM-1 $\beta$-lactamase. In the active domain of TEM-1 $\beta$-lactamase, residues Gly236, Gly238 and Asn170 are located in the periphery region, while Ser235, Arg244, Ala237, Ser70, Ser130 and Asn132 are located in the inside region to form a stable hydrophobic space, namely an active 'pocket' (11). Penicillin $\mathrm{G}$ is an aromatic compound that contains a $\beta$-lactam ring and a hydroxyl group linked to the terminus of the chain-like molecule (46). The $\beta$-lactam ring is located inside the pocket, while the benzene ring on the side chain is located outside the pocket (47). The penicillanate nucleus, 7-C-O, on the $\beta$-lactam ring forms three hydrogen bonds with Ala237, Ser70 and Ser130 of the enzyme. The results from the present study demonstrated that the acetophenone amide group, 1-C-O, on the side chain forms a hydrogen bond with Asn132, while the carbonyl group at position 2 forms two hydrogen bonds with the Arg244 and Ser235 residues of the enzyme. Thus, six amino acids of the enzyme participate in the binding of penicillin $\mathrm{G}$ to TEM-1 $\beta$-lactamase and form a total of 6 hydrogen bonds.

Docking simulations were also performed for cefalexin and cefoxitin using the same method. The results demonstrated that four amino acids of TEM- $1 \beta$-lactamase participated in the binding of cefalexin to TEM-1 $\beta$-lactamase and five hydrogen bonds were formed. For the binding of cefoxitin to TEM-1 $\beta$-lactamase, three amino acids were involved three hydrogen bonds were formed. Therefore, the molecular docking simulation visually presented the binding processes of the three antibiotics to the TEM-1 $\beta$-lactamases, revealing the number of binding sites and the type of binding between the TEM-1 $\beta$-lactamase and the antibiotic substrates, the binding form and strength between the functional groups of the antibiotics and the amino acids of the TEM- $1 \beta$-lactamase.

The energies of the binding complexes of TEM-1 $\beta$-lactamases with penicillin $\mathrm{G}$, cefalexin and cefoxitin were summarized in Table VI. The results in Table VI demonstrated that, during the binding processes, all $\Delta G$ values were negative for each antibiotic, indicating that all binding was spontaneous. Additionally, despite a negligible amount of electrostatic energy $\left(\Delta E_{3}\right)$, hydrogen bonds and Van der Waals energies $\left(\Delta E_{2}\right)$ accounted for nearly the entire intermolecular interaction energy $\left(\Delta E_{1}\right)$ in the binding processes. This demonstrated that the three antibiotics possessed different binding capacities to the TEM-1 $\beta$-lactamase, with penicillin $\mathrm{G}$ possessing the greatest binding capacity and cefoxitin displaying the weakest binding capacity to the enzyme.

The difference in binding capacity was caused by the difference in the structure of the antibiotics. Although penicillin $\mathrm{G}$, cefalexin and cefoxitin all belong to the class of $\beta$-lactam antibiotics, penicillin $\mathrm{G}$ contains a $\beta$-lactam ring that is fused with a thiazolidine ring, while cefalexin contains a $\beta$-lactam ring that is fused with a hydrogenated thiazine ring (48). The three antibiotics all contain a primary amine group that is able to bind to various acyl groups. These acyl groups exhibit marked differences due to different positions and side chain structures (49). The introduction of acyl side chains in different structures may regulate the antibacterial spectrum, the binding mode on the enzyme, the antibacterial activity and the physiochemical properties of the antibiotics (46). Furthermore, the cefaxitin nucleus contains a methoxy group on its 7-C, which blocks the access of the $\beta$-lactam ring and reduces the affinity of the antibiotics to enzymes, and thereby protects the $\beta$-lactam ring from being destroyed (50). Consequently, cefoxitin demonstrated the weakest binding with the TEM-1 $\beta$-lactamase.

In conclusion, recognition and binding of TEM-1 $\beta$-lactamase with three $\beta$-lactam antibiotics, including penicillin $\mathrm{G}$, cefalexin and cefoxitin, was investigated by fluorescence and UV-Vis absorption spectra in combination with molecular docking under simulated physiological conditions. The Stern-Volmer quenching $\left(K_{s v}\right)$ and binding constants $\left(K_{a}\right)$, number of binding sites $(n)$, binding distance $(r)$ and thermodynamic data $(\Delta G, \Delta H$ and $\Delta S)$ were determined at three different temperatures. The results demonstrated that the fluorescence emissions of the TEM-1 $\beta$-lactamase were extinguished by static quenching and the energy was transferred from the enzyme to the antibiotics in a non-radioactive manner. All binding of the TEM-1 $\beta$-lactamase with the three antibiotics was a spontaneously exothermic process without an energy barrier. Furthermore, the binding was driven by enthalpy change and the binding forces between them were predominantly hydrogen bonding and Van der Waals forces. TEM-1 $\beta$-lactamase only bound with one antibiotic at a time with an affinity order of penicillin $\mathrm{G}>$ cephalexin > cefoxitin and the binding capacity between them was closely related to the functional groups and flexibility of the antibiotics. In addition, it was also demonstrated that a conformational change occurred in the TEM-1 $\beta$-lactamases when they bound with the three antibiotics to form a TEM-1-antibiotic complex. Therefore, the present study provided an important insight into the recognition and binding of TEM-1 $\beta$-lactamase with $\beta$-lactam antibiotics, which may be useful for designing a novel substrate for TEM-1 $\beta$-lactamase and developing novel antibiotics resistant to the enzyme. 


\section{Acknowledgements}

The present study was funded by a grant from the National Natural Science Foundation of China (grant no. 21075097).

\section{References}

1. Ji S, Chen Y, Ruan Z, Fu Y, Ji J, Fu Y, Wang H and Yu Y: Prevalence of carbapenem-hydrolyzing class D $\beta$-lactamase genes in Acinetobacter spp. Isolates in China. Eur J Clin Microbiol Infect Dis 33: 989-997, 2014.

2. Li Q, Zhang T and Bian L: Recognition and binding of $\beta$-lactam antibiotics to bovine serum albumin by frontal affinity chromatography in combination with spectroscopy and molecular docking. J Chromatogr B Analyt Technol Biomed Life Sci 1014: 90-101, 2016

3. Tahlan $\mathrm{K}$ and Jensen SE: Origins of the $\beta$-lactam rings in natural products. J Antibiot (Tokyo) 66: 401-410, 2013.

4. Cho H, Uehara T and Bernhardt TG: Beta-lactam antibiotics induce a lethal malfunctioning of the bacterial cell wall synthesis machinery. Cell 159: 1300-1311, 2014.

5. Vasoo S, Barreto JN and Tosh PK: Emerging issues in gram-negative bacterial resistance: An update for the practicing clinician. Mayo Clin Proc 90: 395-403, 2015.

6. Martínez-Martínez L and González-López JJ: Carbapenemases in Enterobacteriaceae: Types and molecular epidemiology. Enferm Infecc Microbiol Clin 32 (Suppl 4): S4-S9, 2014.

7. Porres-Osante N, Sáenz Y, Somalo S and Torres C: Characterization of beta-lactamases in Faecal Enterobacteriaceae recovered from healthy humans in Spain: Focusing on AmpC polymorphisms. Microb Ecol 70: 132-140, 2015.

8. Becciro A, Mabarjan S, Gaulton T, Doumith M, Soares NC, Dhanji H, Warner M, Doyle M, Hickey M, Downie G, et al False extended-spectrum \{beta\}-lactamase phenotype in clinical isolates of Escherichia coli associated with increased expression of OXA-1 or TEM-1 penicillinases and loss of porins. J Antimicrob Chemother 66: 2006-2010, 2011.

9. Bush K and Jacoby GA: Updated functional classification of beta-latamases. Antimicrob Agents Chemother 54: 969-976, 2010 .

10. Singh MK and Dominy BN: The evolution of cefotaximase activity in the TEM $\beta$-lactamase. J Mol Biol 415: 205-220, 2012.

11. Levitt PS, Papp-Wallace KM, Taracila MA, Hujer AM, Winkler ML, Smith KM, Xu Y, Harris ME and Bonomo RA: Exploring the role of a conserved class A residue in the $\Omega$-Loop of KPC-2 $\beta$-lactamase: A mechanism for ceftazidime hydrolysis. J Biol Chem 287: 31783-31793, 2012.

12. Bös F and Pleiss J: Multiple molecular dynamics simulations of TEM beta-lactamase: Dynamics and water binding of the omega-loop. Biophys J 97: 2550-2558, 2009.

13. Salverda ML, De Visser JA and Barlow M: Natural evolution of TEM-1 $\beta$-lactamase: Experimental reconstruction and clinical relevance. FEMS Microbiol Rev 34: 1015-1036, 2010.

14. Krizova L, Poirel L, Nordmann P and Nemec A: TEM-1 $\beta$-lactamase as a source of resistance to sulbactam in clinical strains of Acinetobacter baumannii. J Antimicrob Chemother 68: 2786-2791, 2013

15. Wang J, Palzkill T and Chow DC: Structural insight into the kinetics and DeltaCp of interactions between TEM-1 beta-lactamase and beta-lactamase inhibitory protein (BLIP). J Biol Chem 284: 595-606, 2009

16. Legendre D, Vucic B, Hougardy V, Girboux AL, Henrioul C, Van Haute J, Soumillion P and Fastrez J: TEM-1 beta-lactamase as a scaffold for protein recognition and assay. Protein Sci 11: 1506-1518, 2002.

17. Hanes MS, Reynolds KA, McNamara C, Ghosh P, Bonomo RA, Kirsch JF and Handel TM: Specificity and cooperativity at $\beta$-lactamase position 104 in TEM-1/BLIP and SHV-1/BLIP interactions. Proteins 79: 1267-1276, 2011.

18. Cheong WL, Tsang MS, So PK, Chung WH, Leung YC and Chan PH: Fluorescent TEM-1 $\beta$-lactamase with wild-type activity as a rapid drug sensor for in vitro drug screening. Biosci Rep 34: pii:e00136, 2014.

19. Phichith D, Bun S, Padiolleau-Lefevre S, Guellier A, Banh S, Galleni M, Frere JM, Thomas D, Friboulet A and Avalle B: Novel peptide inhibiting both TEM-1 $\beta$-lactamase and penicillin-binding proteins. FEBS J 277: 4965-4972, 2010.
20. Mathieu V, Fastrez J and Soumillion P: Engineering allosteric regulation into the hinge region of a circularly permuted TEM-1 beta-lactamase. Protein Eng Des Sel 23: 699-709, 2010.

21. Watkins RR, Papp-Wallace KM, Drawz SM and Bonomo RA: Novel $\beta$-lactamase inhibitors: A therapeutic hope against the scourge of multidrug resistance. Front Microbiol 4: 392 , 2013.

22. Patel AB, Khumsupan P and Narayanaswami V: Pyrene fluorescence analysis offers new insights into the conformation of the lipoprotein-binding domain of human apolipoprotein $\mathrm{E}$. Biochemistry 49: 1766-1775, 2010.

23. Erez Y, Simkovitch R, Shomer S, Gepshtein R and Huppert D: Effect of acid on the ultraviolet-visible absorption and emission properties of curcumin. J Phys Chem A 118: 872-884, 2014.

24. van de Weert M and Stella L: Fluorescence quenching and ligand binding: A critical discussion of a popular methodology. J. Mol Struct 998: 144-150, 2011.

25. Stoddard BL and Koshland DE Jr: Prediction of the structure of a receptor-protein complex using a binary docking method. Nature 358: 774-776, 1992.

26. Sarkar M, Paul SS and Mukherjea KK: Interaction of bovine serum albumin with a psychotropic drug alprazolam: Physicochemical, photophysical and molecular docking studies. J Lumin 142: 220-230, 2013.

27. Tiwari R, Mahasenan K, Pavlovicz R, Li C and Tjarks W: Carborane clusters in computational drug design: A comparative docking evaluation using AutoDock, FlexX, Glide, and Surflex. J Chem Inf Model 49: 1581-1589, 2009.

28. DeLano WL: The PyMOL molecular graphics system. DeLano scientific, San Carlos, CA, USA, 2002.

29. Soonwoo Chah and Matthew R: Hammond and Richard N: Zare gold nanoparticles as a colorimetric sensor for protein conformational changes. Chem Biol 12, 323-328, 2005.

30. Chen W, Habibul N, Liu XY, Sheng GP and Yu HQ: FTIR and synchronous fluorescence heterospectral two-dimensional correlation analyses on the binding characteristics of copper onto dissolved organic matter. Environ Sci Technol 49: 2052-2058, 2015.

31. Pannipara M, Asiri AM, Alamry KA, Arshad MN and El-Daly SA: Spectroscopic investigation, effect of solvent polarity and fluorescence quenching of a new D- $\pi$-A type chalcone derivative. J Fluoresc 24: 1629-1638, 2014.

32. Zhang G, Wang A, Jiang $\mathrm{T}$ and Guo J: Interaction of the irisflorentin with bovine serum albumin: A fluorescence quenching study. J Mol Struct 891: 93-97, 2008

33. Lakowicz JR: Principles of fluorescence spectroscopy, 3rd (eds), Springer Press, New York, pp278, 2006.

34. Lakos Z, Szarka Á, Koszorús L and Somogyi B: Quenching-resolved emission anisotropy: A steady state fluorescence method to study protein dynamics. J Photoch Photobio B Biol 27: 55-60, 1995

35. Weng $\mathrm{C}, \mathrm{Fu} \mathrm{Y}$, Jiang $\mathrm{H}$, Zhuang $\mathrm{S}$ and $\mathrm{Li} \mathrm{H}$ : Binding interaction between a queen pheromone component HOB and pheromone binding protein ASP1 of Apis cerana. Int J Biol Macromol 72: 430-436, 2015

36. Ross PD and Subramanian S: Thermodynamic of protein association reactions: Forces contributing to stability. Biochemistry 20: 3096-3102, 1981.

37. Deng Y and Roux B: Computation of binding free energy with molecular dynamics and grand canonical Monte Carlo simulations. J Chem Phys 128: 115103, 2008.

38. Stewart MH, Huston AL, Scott AM, Oh E, Algar WR, Deschamps JR, Susumu K, Jain V, Prasuhn DE, Blanco-Canosa J, et al: Competition between Förster resonance energy transfer and electron transfer in stoichiometrically assembled semiconductor quantum dot-fullerene conjugates. ACS Nano 7: 9489-9505, 2013.

39. Xie Y, Yang X, Pu J, Zhao Y, Zhang Y, Xie G, Zheng J, Yuan H and Liao F: Homogeneous competitive assay of ligand affinities based on quenching fluorescence of tyrosine/tryptophan residues in a protein via Főrster-resonance-energy-transfer. Spectrochim Acta A Mol Biomol Spectrosc 77: 869-876, 2010.

40. Liao F, Xie Y, Yang X, Deng P, Chen Y, Xie G, Zhu S, Liu B, Yuan H, Liao J, et al: Homogeneous noncompetitive assay of protein via Förster-resonance-energy-transfer with tryptophan residue(s) as intrinsic donor(s) and fluorescent ligand as acceptor. Biosens Bioelectron 25: 112-117, 2009.

41. Ye Z, Ying Y, Yang X, Zheng Z, Shi J, Sun Y and Huang P: A spectroscopic study on the interaction between the anticancer drug erlotinib and human serum albumin. J Incl Phenom Macro Chemist 78: 405-413, 2014. 
42. Mustafi D, Sosa-Peinado A and Makinen MW: ENDOR structural characterization of a catalytically competent acylenzyme reaction intermediate of wild-type TEM-1 beta-lactamase confirms glutamate-166 as the base catalyst. Biochemistry 40 2397-2409, 2001.

43. Christov C, Ianev D, Shosheva A and Atanasov B: pH-dependent quenching of the fluorescence of tryptophan residues in class A beta-lactamase from E. coli (TEM-1). Z Naturforsch C 59: 824-827, 2004

44. Liu Q, Grant G and Vo-Dinh T: Investigation of synchronous fluorescence method in multicomponent analysis in tissue. IEEE J Sel Top Quantum Electron 16: 927-940, 2010.

45. Miller JN: Recent advances in molecular luminescence analysis. Anal Proc 16: 203-209, 1979.

46. Hubschwerlen C: $\beta$-latam antibiotics. Comprehensive Med Chemistry II: 479-451, 2007.
47. Suárez C and Gudiol F: Beta-lactam antibiotics. Enferm Infecc Microbiol Clin 27: 116-129, 2009 (In Spanish).

48. Endo R, Yamane N, Tamayose MH, Uchibori KK and Nakasone I: A simple disk diffusion test to identify beta-lactamase-negative, ampicillin-resistant Haemophilus influenzae-application of cephalexin, cefsulodin and cefaclor disks. Rinsho Byori 58: 963-971, 2010 (In Japanese).

49. Tahlan $\mathrm{K}$ and Jensen SE: Origins of the $\beta$-lactam rings in natural products. J Antibiot (Tokyo) 66: 401-410, 2013.

50. Rubach JK, Cui G, Schneck JL, Taylor AN, Zhao B, Smallwood A, Nevins N, Wisnoski D, Thrall SH and Meek TD: The amino-acid substituents of dipeptide substrates of cathepsin C can determine the rate-limiting steps of catalysis. Biochemistry 51: 7551-7568, 2012. 